\title{
Historia De La Universidad Pública Latinoamericana: Una Visión General
}

\author{
César Ernesto González Coronado \\ Liliana Ibeth Castañeda Rentería \\ Universidad de Guadalajara, México
}

Doi: 10.19044/esj.2018.v14n4p104 URL:http://dx.doi.org/10.19044/esj.2018.v14n4p104

\begin{abstract}
This text describes on a synthetic way the history that characterizes the development of public Latin America's universities. Through an intense literature review three core aspects are identified: enrollment generalization, the growth of private institutions and the politicization within the processes, considered as an essential part of the public universities in the continent. The analysis states that even if they are not negative, these characteristics are by themselves product of a history loaded by the social mobility and by the citizenship formation.
\end{abstract}

Keywords: Generalization, politicization, private higher education, public universities

\section{Resumen}

La presente comunicación describe de manera sintética la historia que ha caracterizado el desarrollo de las universidades públicas latinoamericanas. A través de una revisión bibliográfica se identifican tres aspectos centrales: la masificación de la matrícula, el incremento de las instituciones de educación superior privadas y los procesos de politización que han impreso su esencia en la mayoría de universidades públicas en nuestro continente. El análisis indica que dichas características aunque no siempre negativas, son en sí mismas producto de una historia que ha dejado en estas instituciones la carga de la movilidad social y la formación de ciudadanía.

Palabras-clave: Universidad pública, masificación, politización y educación superior privada 
"Todas las grandes estructuras universitarias modernas pueden ser definidas como productos residuales de la vida de sus pueblos" (Darcy Ribeiro).

Sin duda todos reconocemos que como producto social, las instituciones tienden a reproducir las condiciones del medio social en que están enclavadas, también es cierto que estas instituciones logran cuajar un alma propia, generada por la organización adoptada o por las características en las que se ve obligada a desenvolverse, lo cual les permite no solo ser resultado, sino también convertirse algunas veces en protagonistas de acontecimientos sociales.

Si bien esto suena lógico, valdría la pena preguntarnos si como sociedad somos capaces de reconocer a la universidad como producto de una historia particular, y si hemos sido capaces de apreciar en su justa dimensión las características heredadas de dicha historia; se trata así de extender la mirada y ver en la historia social en la que está enclavada la Universidad latinoamericana no sólo el obstáculo, sino también la riqueza particular para su desarrollo futuro.

El presente trabajo pretende no ser un relato más de lo que autores como Levy, Brunner, Latapí u otros, han planteado en sus respectivos trabajos, sino que a partir de integrar sus planteamientos, pretendemos construir un análisis que nos permita una adecuada comprensión de lo que es hoy nuestra universidad. Se toman en cuenta características comunes en la mayoría de países latinoamericanos, sin dejar de estar conscientes de la heterogeneidad de nuestras realidades.

El trabajo será desarrollado en seis apartados. El primero que comprende algunos de los antecedentes históricos más importantes de nuestra universidad, que servirá como preámbulo al análisis. A partir de ahí se presentan acontecimientos tales como la masificación, la politización y la privatización de la universidad latinoamericana respectivamente. Le sigue una recapitulación, y al final una conclusión presentando algunos de los retos y compromisos de la universidad actual.

Se hace necesario establecer de manera textual, las limitaciones del presente trabajo. Si bien es cierto que se eligieron tres temáticas que caracterizan a la universidad latinoamericana, de manera arbitraria, sería deshonesto aseverar que son las únicas o las más importantes. Digamos así que la elección fue motivada por intereses personales de los autores lo cual no implica que pensemos que la masificación, la politización, así como el incremento de la presencia de instituciones de educación superior privadas sean las únicas características que configuran la identidad de las universidades latinoamericanas. Reconocemos también por ejemplo, la triple misión universitaria: docencia, investigación y extensión, así como la demanda de los 
sectores sociales y privados de generar conocimiento "útil" y generador de riqueza, el trabajo que se realiza para la internacionalización, entre otros aspectos que caracterizan hoy la universidad pública del continente. Aclarado esto, demos inicio con el desarrollo del tema.

\section{Antecedentes}

Algo que ha caracterizado la historia latinoamericana, ha sido la costumbre o la tendencia casi, podríamos decir, natural a imitar o copiar modelos de otros países que consideramos avanzados.

Debemos reconocer que esta característica es producto de una historia tan lejana como la propia conquista americana. La religión, el gobierno, costumbres e instituciones fueron traídas del viejo continente a un contexto por mucho diferente al original, que con el tiempo les daría características propias. Un claro ejemplo lo tenemos en la Universidades, las cuales fueron una copia de la Universidad de Salamanca importada al nuevo continente.

No se sabe a ciencia cierta cuál fue la primera universidad de América; si fue la de Lima o la de México (Silva-Herzog, 1999:1) ${ }^{4}$, pero ambas instituciones tenían como encomienda la transmisión del conocimiento y la formación de las élites políticas y dirigentes.

En el caso de la Real y Pontificia Universidad de México, alumnos criollos y de origen europeo, recibían cátedras de filosofía, literatura, medicina y lenguas indígenas. En poco tiempo la enseñanza impartida en esta casa de estudios alcanzó fama en Europa como vanguardia de la educación superior en América Latina (Pallán, 1994:11). Como se podrá observar la universidad nace como una institución esencialmente dedicada a la enseñanza, a la formación profesional en las áreas tradicionales como la medicina y la abogacía (Kent, 1996:10).

Otra característica de la Universidad colonial fue sin duda la simbiosis entre el Estado y la Iglesia (Ibid :11), simbiosis que los países hispanoamericanos disolvieron con su independencia y que se expresó por medio de la secularización de la educación superior.

Es a partir de este momento que la universidad latinoamericana empieza a escribir con tinta propia su historia, aunque sin poder librarse del todo de la sombra de la imitación, ya que a pesar de la independencia, las universidades latinoamericanas, eran fiel reflejo de las estructuras sociales que esta lucha no había logrado modificar, seguían siendo los "virreinatos del espíritu" y conservaban su carácter de academias señoriales (Tünnermann, $1997: 5)$.

\footnotetext{
${ }^{4}$ Existe una confusión dado que la Cédula Real que creó la Universidad Mayor de San Marcos de Lima fue fechada el 12 de mayo de 1551, en tanto que la de México tiene como fecha el 21 de Septiembre del mismo año; sin embargo la Real Universidad de México, comenzó sus cátedras años antes que la limeña .
} 
Históricamente la universidad está marcada por dos luchas: la autonomía universitaria y la participación de los estudiantes en el gobierno de las instituciones (Kent, 1996:14) ambas claramente identificadas en el movimiento de Córdoba de 1918. El Movimiento de Córdoba, es considerado por autores como Tünnermann, "la primera confrontación entre una sociedad, que comenzaba a experimentar cambios de su composición interna y una Universidad enquistada en esquemas obsoletos" (1997:6).

Otra característica en nuestro contexto ha sido la frecuente oposición política e ideológica al Estado por parte de la universidad pública (Levy, 1995:497). Durante mucho tiempo la Universidad fue objeto de múltiples clausuras y aperturas, decisión que se tomaba dependiendo del grupo en el poder para el cual representara un apoyo o en su caso una oposición.

Según Roberto Rodríguez Gómez (2000:19) es a partir de los años sesenta que las instituciones de enseñanza superior en América Latina desarrollaron procesos de crecimiento, diversificación y reforma, debido esto tal vez, al crecimiento demográfico ocurrido en Latinoamérica.

Analicemos algunos puntos específicos de la historia de la Universidad Latinoamericana que definieron en mucho su personalidad: la masificación, la politización y la privatización de la matrícula.

\section{Masificación}

Históricamente el fenómeno de masificación de la Educación superior tuvo lugar en Latinoamérica de manera asincrónica y desordenada, es decir, se llevó a cabo sin contar con una plataforma adecuada (Rodríguez Gómez: 20), sin haber consolidado la escolarización de los niveles primaria y secundaria.

Además, surge con ella una gran paradoja (Brunner, 1987: 39): la universidad se masificó contra el mercado. Contrariamente a lo que se pensó, a partir de la adopción por parte de los estados del concepto de modernización, que supusieron sería el camino hacia el desarrollo, las políticas tendientes al fortalecimiento de la educación superior en relación con las demandas del mercado no tuvieron los resultados esperados.

En contra de lo previsto, la matrícula se expandió en las áreas de ciencias sociales y humanidades, convirtiéndose así la universidad, en una herramienta básica de movilidad social de las clases media y media baja, pero con un débil vínculo con el sector industrial, tecnológico y de servicios.

La universidad latinoamericana se desarrolló en un ambiente "populista", con una gran carga de responsabilidad social, por lo que fue imposible generar condiciones que produjeran o apoyaran una ideología de mercado, esto a pesar de que la universidad ha sido vista, al menos en el discurso, como fundamental en el desarrollo nacional, aspecto que al parecer se ve influenciado por el carácter social que se le ha dado a esta institución. 
Brunner (1987:75) propone una hipótesis respecto a los procesos que favorecieron la masificación:

- La matrícula crece en parte impulsada por la incorporación de la mujer a la enseñanza superior;

- La matrícula crece en parte como resultado de la diversificación institucional de la enseñanza superior;

- La matrícula crece, punto relacionado con el inmediatamente anterior, en parte como producto de la regionalización de la matrícula.

Por supuesto, la expansión de la matrícula no fue un problema aislado. La necesidad a la que se enfrentó el Estado fue la de aumentar la cantidad de docentes para poder hacer frente a la expansión de la matrícula, lo que ocasionó que no pocas veces se incluyera a profesionales con ninguna experiencia docente y comúnmente recién egresados, lo que Kent llama una planta académica improvisada (1998: 314).

\section{Politización}

Una de las características que ha distinguido a la comunidad universitaria latinoamericana es la sólida participación política que ha tenido, ya sea en relación con el estado, en relación con partidos políticos, grupos de presión y por supuesto alrededor de los grupos de interés que se desenvuelven en su vida interna.

Las raíces de la politización pueden ser rastreadas hasta las reformas de 1918 (Levy, 1995: 121). Debemos aclarar también que la politización no es un fenómeno exclusivo de las instituciones latinoamericanas, sin embargo, ha sido en nuestro continente donde ha alcanzado, según lo expresa Brunner (1987: 25), caracteres de naturaleza e intensidad propios.

Decíamos ya que una de las características distintivas de nuestras universidades es la participación de los estudiantes en el gobierno de estas, esto le ha redituado una importancia extra, en la medida en que otros actores -gremios o partidos políticos, por ejemplo- no siempre han podido llegar a organizarse (Levy, 1995: 124).

De igual manera, la influencia de la organización estudiantil, en algún tiempo más de izquierda, exigían que la universidad no sólo se comprometiera a la transmisión de conocimiento, sino ocupara un papel estratégico y de vanguardia en la transformación social y en lo posible, en la revolución y la liberación nacional (Brunner, 1987: 25).

Una de las problemáticas centrales en el ámbito político de la universidad, ha sido desde luego el debate respecto a los significados y alcances del concepto de autonomía, además de la incapacidad estatal para evitarlo. 
Existen tres principios que constituyen la sustancia misma de la autonomía, mismos que son intrínsecos e inseparables de la naturaleza de la universidad (Reséndiz, 2000: 67)

a) Cada universidad debe ser objetivamente una comunidad, es decir, una congregación cuyos intereses comunes dominen o prevalezcan de manera natural sobre toda la gama de intereses particulares, específicos o peculiares de cada individuo o grupo integrante de la congregación.

b) En todo lo que no pertenezca al ámbito de los intereses comunes propios de una congregación universitaria, los integrantes de ésta se rigen por las leyes y normas generales del estado. Las diferencias de opinión o posición relativas a asuntos ajenos a los intereses comunes no se dilucidan necesariamente dentro de la institución, y los asuntos pertenecientes al ámbito de la comunidad universitaria se debaten y resuelven en el seno de esta y con exclusión expresa de la coerción física.

La tradición universal marcaría el apego de cualquier universidad a estos tres principios, sin embargo, en Latinoamérica las condiciones de financiamiento, y los diferentes eventos sociales y políticos de nuestros países, han convertido a la universidad en víctima de su propia autonomía, o, mejor dicho, de lo que el dirigente en turno piense o conceptualice como tal.

Como consecuencia de la politización surge lo que Mario Albornoz llama "la universidad moderna" ( in fecha) cuya identidad se forja en torno a su triple misión: la docencia, la investigación y la extensión. Esto imprimió el compromiso social de las universidades públicas latinoamericanas, con excepción de Brasil.

\section{Privatización de la matrícula}

Se hace necesario precisar la connotación que le estamos dando en el presente trabajo al término privatización, no como forma de financiamiento por parte de particulares, sino la expansión de la matrícula que han experimentado instituciones de educación superior privada en las últimas décadas. Veamos primero este último.

La historia de la privatización de la matrícula, se plantea en tres olas (Levy, 1995:80); la primera caracterizada por el surgimiento de universidades coloniales, mezcla de instituciones públicas y privadas; la segunda, con el surgimiento del monopolio público; la tercera, con el surgimiento de universidades privadas propiamente dichas.

Por su parte las universidades privadas dividen su desarrollo en tres olas, la primera con el surgimiento de las instituciones religiosas, la segunda, caracterizada con el surgimiento de universidades privadas seculares de élite, y la tercera, cuyo sello distintivo es el surgimiento de instituciones privadas y seculares de absorción de la demanda (Ibid). 
A manera de hipótesis, podríamos establecer que la expansión de la matrícula privada en Latinoamérica se debe en primera instancia al fracaso de la Universidad pública al no ser capaz de mantener el grado de diferenciación que las clases de elite demandaban, según lo establecen autores como Levy (99:1995), dado el fenómeno de masificación que tuvo lugar en las instituciones de educación superior, no solo por el crecimiento de la población, sino también por el desarrollo económico. Las élites ya no veían representados sus intereses en universidades repletas de alumnos de clases sociales inferiores a la propia.

Este no fue el único "fracaso" de la universidad pública que propicio de alguna manera el desarrollo y fortalecimiento del sector privado, también está el político y el económico. La politización significó en más de una ocasión un peligro para las clases dominante, así como inestabilidad en las universidades públicas; respecto al fracaso económico, tenemos el hecho de la mala administración de los recursos otorgados a las instituciones. La universidad pública ya no era un espacio que ofreciera un status de diferenciación para la élite.

Sin embargo, el surgimiento del sector privado no solo se puede explicar como consecuencia de las fallas en el sector público, sino que se hace necesario identificar a los actores involucrados en la creación de alternativas privadas (Ibid:113). Entre estos actores encontramos a las propias instituciones, a los alumnos con mayor capacidad de elección, y por supuesto al mercado.

Así mismo encontramos el cambio de actores que en la escena educativa han aparecido a través de la historia. En un principio, nos dice Durham (2000:83), la tensión en la educación superior privada era entre el Estado, la comunidad académica y la iglesia; en la actualidad la tensión se establece entre el Estado y el mercado.

El mercado ha sido uno de esos actores invisibles pero omnipresentes en la vida de las instituciones de educación superior tanto públicas como privadas. En lo que respecta a las instituciones privadas, establece García de Fanelli y J. Balán (1996:12), este sector está más atento a las demandas fluctuantes del mercado. De este modo, el comercio juega naturalmente un papel visible en la fundación y el financiamiento de las universidades privadas (Levy, 1995:128) y ofrece una ventaja para los egresados de este tipo de instituciones.

Además de esta ventaja, el sector privado ofrece una estabilidad política y administrativa más sólida que una universidad pública. Como ejemplo se podría hablar de la duración de rectorados, o de la gobernabilidad en algunas instituciones. En suma, factores económicos, políticos y de clase social han provisto razones específicas para el desarrollo del sector privado, 
basado particularmente en los "fracasos", diría Levy, del sector público (Ibid: 130) y el descontento y falta de calidad (Durham, 2000: 96).

Durham, nos presenta por su parte tres periodos del desarrollo de la educación superior privada a partir de 1950 (ver tabla 1).

\begin{tabular}{|c|c|}
\hline $1955-1965$ & $\begin{array}{c}\text { En el transcurso de ese periodo, con excepción de Argentina y con } \\
\text { algunas variantes coyunturales, lo que se observa es una expansión } \\
\text { paralela de los sectores públicos y privados, con tendencia a } \\
\text { mantener el equilibrio relativo entre ambos. }\end{array}$ \\
\hline $1965-1975$ & $\begin{array}{c}\text { Debilitamiento de la universidad pública (originado por la } \\
\text { incapacidad de absorber la demanda y la pérdida de prestigio entre } \\
\text { las élites). }\end{array}$ \\
\hline $1980-1990$ & $\begin{array}{c}\text { Los años ochenta inauguran una nueva fase para los sistemas de } \\
\text { educación superior en los países latinoamericanos. Las razones en } \\
\text { ese marco referencial pueden ordenarse alrededor de dos ejes: } \\
\text { La consolidación de la participación ampliada de la educación } \\
\text { superior privada, y } \\
\text { La redefinición, en los diferentes países, de la relación del estado con } \\
\text { sus respectivos sistemas nacionales de ES. }\end{array}$ \\
\hline
\end{tabular}

Tabla 1. Características de los periodos evolutivos de la educación superior privada, según Durham (200:93-102).

En los últimos años podemos ver que el universo de instituciones de educación superior privadas se ha incrementado y presenta además una diversidad importante en cuanto a la calidad educativa. Pero además coexisten en Latinoamérica "una pluralidad de instituciones de educación superior, universitarias, no universitarias, públicas y privadas, instituciones de excelencia orientadas a la investigación y al posgrado, e instituciones orientadas a la docencia y a la educación de grado" (OEI, 2014)

Hasta aquí hemos presentado de manera general tres de los elementos característicos de la historia de las universidades latinoamericanas que si bien no son los únicos, pensamos son los que determinan en mayor medida la identidad de las universidades latinoamericanas. A continuación se presenta una recapitulación de lo que estos acontecimientos han significado para la vida universitaria.

\section{Recapitulación}

La universidad como instrumento de movilidad social (que las clases medias identificaron como una oportunidad), ha sido blanco de muchos intereses, que no siempre han apoyado a su fortalecimiento y pertinencia.

Como ejemplo de ello tenemos algunos aspectos de la masificación de la matrícula, desarrollo cuantitativo que estuvo impulsado por motivaciones básicamente de tipo social y político (Villaseñor, 1997:20), habiéndose descuidado aspectos cualitativos como el de proceso enseñanza aprendizaje y el vínculo universidad- mercado. 
En este mismo sentido, autores como Kent (2000: 307), se muestran escépticos ante conceptos como el de masificación, argumentando que no se puede hablar en la actualidad de una educación superior de masas, cuando observamos el gran rezago que en esta materia seguimos teniendo.

Los estudiantes proclamaban que la función más importante de la universidad no era la de proporcionar educación, sino la de cambiar la sociedad (Levy, 1995 : 127). Valdría la pena cuestionar si la identidad universitaria revolucionaria, socialista, comprometida con su entorno ha sobrevivido al embate de la realidad, y hasta donde esta institución tiene para sus integrantes un valor más allá de la credencial que otorga.

Por otro lado Tünnermann (1997: 7), menciona que la problemática universitaria latinoamericana no puede ser entendida, en su verdadera naturaleza y complejidad, sin un análisis de lo que significa la Reforma de Córdoba.

Los grandes logros siempre mencionados de dicha reforma son sin duda, el cogobierno (en el que los estudiantes logran participar) y la autonomía, logros que más tarde serían viciados por intereses partidistas, en el caso de la participación estudiantil, y víctimas de interpretaciones no siempre claras sobre el concepto de la autonomía, sin olvidar la represión de algunos gobiernos.

Según Luis Manuel Peñalver, "la Reforma de Córdoba de 1918 fue revolucionaria para su tiempo: cambió la estructura y las relaciones de poder universitario, echó por tierra los dogmas filosóficos, liberó a la universidad del sectarismo religioso heredado de la Colonia y abrió las puertas de aquélla a los sectores mayoritarios que se incorporaban políticamente en el sistema de democracia liberal, pero no llegó a cambiar la orientación de fondo de la Universidad ni a adaptar su labor al proceso de desarrollo de nuestros pueblos" (Ibid: 86).

En la actualidad la universidad pública latinoamericana, se enfrenta a un desprestigio y a una falta de confianza por parte de la sociedad, producto de acontecimientos sociales, políticos y al bombardeo constante de organismos internacionales. Por otro lado tenemos que el sector privado de educación superior, se ha expandido de manera impresionante y lo ha hecho gracias a la despreocupación del Estado por garantizar una educación superior de calidad y bajo la sombra "inminente" de las propias universidades.

Aquí resulta indispensable señalar que si bien es preocupante el crecimiento casi desmedido de instituciones privadas de educación superior y la falta de calidad en algunas de las universidades privadas (sobre todo de tercera generación de instituciones privadas), lo alarmante es el desinterés del estado, por un lado, y por otro la despreocupación de las propias universidades públicas para crear las políticas y lograr adaptarse a una realidad que les 
demanda no solo mayor calidad, sino también un verdadero compromiso con el desarrollo nacional.

\section{Conclusion}

Tal como lo cita Clark (1983:113) “en cualquier situación en que algún grupo humano viva en común y relativamente aislado del resto de la gente, en un rincón social compartido, con problemas y quizás con enemigos en común, emerge la cultura". Cultura que a su vez está compuesta de subculturas producto natural de las organizaciones.

Las universidades cuentan con la subcultura académica, la estudiantil y la administrativa (Ibid), y además cada una de estas subculturas, en el caso de Latinoamérica ha creado una identidad que no ha logrado insertarse de manera eficiente en las condiciones de nuestra realidad.

La apreciación de Luis Alberto Sánchez es interesante, "a pesar de tantos años (...) América (Latina) esta aferrada (...) al entreguismo, a la imitación servil (...) al divorcio entre la inteligencia y el pueblo, y entre la inteligencia y la Universidad" (Tünnermann, 1997:87). Interesante, no porque sea o no cierto, sino porque eso nos han hecho creer, o mejor dicho, eso nos han dicho para que dejemos de creer en la universidad.

En la última década se ha configurado sobre la universidad, además de las misiones de formar profesionales, ciudadanos, extender la cultura y generar conocimiento, el mandato sobre "el uso, la aplicación y explotación” de dicho conocimiento. Lo anterior genera tensiones pues al dotar de valor económico a los productos de investigación, la formación de ciudadanía, la extensión de la cultura, pasan a ser actividades no valoradas y exige a su vez que la universidad se convierta en "un actor decisivo en los procesos de desarrollo social y económico, a través de una vinculación mucho más estrecha con los diferentes agentes de su entorno" (Albornoz, 2014: 54). Esta demanda a la universidad puede ser considerada en la actualidad como otra de las características de la universidad latinoamericana.

La universidad en América Latina, en el marco del paradigma geocultural $^{5}$, dice Yus (Herrera, 2004) requiere de un proyecto universitario que anteponga la ética, el humanismo, la ciencia y la cultura a las estrictas reglas del mercado y de la economía. Esto implica que el modelo educativo deberá preocuparse por la formación de profesionales preocupados por su

5 Para Gorostiaga (1999), esta función de la universidad pública es lo que asegura su coparticipación en un cambio de época, denominado por él como de Paradigma geocultural, caracterizado por impulsar la presencia de nuevos actores (ciudadanía, sociedad civil, género, cultura, ambiente, educación) y nuevas sinergias (Estado-mercado-sociedad civil). El paradigma geocultural requiere la promoción de un pensamiento articulado por cuatro ejes: desarrollo humano sostenible, formación valoral, identidad y multiculturalidad. 
entorno, su cultura y con una base sólida de valores, que les permita ser críticos pero a la ves propositivos.

El quehacer universitario, menciona María Teresa Yurén Camarena (1997: 206), no debe estar subordinado a la creación de la ciencia como su fin último, sino que la universidad debe ser la mediación gracias a la cual la ciencia sirve a la intención de dignificar la vida de la sociedad.

Se debe buscar una "Universidad" social, pero no en el sentido que se ha venido acarreando desde hace décadas, sino como una universidad comprometida académica y científicamente con el bienestar y el desarrollo de comunidad local pero consciente de la influencia global en que vivimos.

La realidad que viven actualmente las universidades latinoamericanas no es solo responsabilidad suya, el contexto de inestabilidad política que no pocas veces enfrentaron países latinoamericanos, así como las crisis financieras, han hecho de estas instituciones víctimas de una realidad áspera.

Después de todo, menciona Acosta, "las universidades son, en cierta forma, no solo creaturas de una sociedad en movimiento, sino también, y acaso esencialmente, instituciones que contribuyen de una u otra forma a que esa sociedad se reconozca a sí misma en los espejos que la propia universidad construye con su desempeño" (2000:334).

Se vuelve prioritario en este contexto el promover estudios que analicen la historia de la universidad, desde una perspectiva analítica que nos permita desdoblar su secuencia genética y así comprender mejor muchos de los rasgos y enfermedades que hoy están presentes en nuestras instituciones. No podemos seguir avanzando sin voltear y reconocer lo que fuimos y lo que dejamos atrás, y más aún, no podemos avanzar con una carga tan pesada como una historia inconclusa.

Los retos son muchos, y el futuro no muy prometedor, ya que en este reto la institución no va sola, necesita al Estado, pero no como fiscalizador, sino como aliado con capacidad de definir un proyecto nacional en el que se fortalezca y aprecie la función de la universidad pública.

\section{References:}

1. Acosta, A. (2000) Estado, políticas y universidades en un periodo de transición, Guadalajara, México: UdeG/ FCE.

2. Albornoz, M. (2014) Presentación: La universidad latinoamericana en debate, en Revista Iberoamericana de Ciencia, Tecnología y Sociedad, Vol. 9, número 27, pp. 49-61.

3. Clark, R. (1983) El sistema de educación superior. Una visión comparativa de la organización académica, México: UAM-A, Editorial Patria.

4. Durham, E. y Sampaio, H. (2000) "La Educación privada en América Latina: estado y mercado" en Balán, J. (coord.), Políticas de reforma 
de la educación superior y la universidad latinoamericana hacia el final del milenio, México: CRIM-UNAM.

5. García de Fanelli, A. y Balán, J. (2000) "El sector privado de la Educación Superior” en Kent, R. (comp.) (1996-1997) “Los temas críticos de la educación superior en América Latina. Estudios comparativos" (2 vols.),México: FCE/ UAA/ FLACSO.

6. Herrera, A. (2004)'La Universidad pública: entre la modernidad y el desarrollo humano" en Reencuentro no 40. http://www.redalyc.org/articulo.oa?id=34004008 (consultada el 12 de febrero de 2018).

7. Kent, R. (2004) en Latapí Sarre, Pablo. (2004). "Un siglo de educación en México" Vol. II. México: FCE, Consejo Nacional Para la Cultura y las Artes. 2a. Reimpresión

8. Levy, D. (1995) La educación Superior y el Estado en Latinoamérica. Desafios privados al predominio público, México: UNAM, FLACSO, Editorial Miguel Angel Porrúa.

9. Organización de Estados Iberoamericanos, OEI (2014), Ciencia, tecnología e innovación para el desarrollo y la cohesión social. Madrid: Programa iberoamericano en la década de los bicentenarios.

10. Pallán Figueroa, C. (1978) Bases para la administración de la educación superior en América Latina: el caso de México, México: INAP.

11. Pallán Figueroa, C., et. Al. (1994) La Educación Superior en México, México: ANUIES.

12. Reséndiz Núñez, D. (2000) Futuros de la Educación Superior en México, México: Siglo XXI.

13. Rodríguez Gómez, R. (2000) “Educación superior y desarrollo en América Latina. Un ensayo de interpretación" en Balán, Jorge (coord.) Políticas de reforma de la educación superior y la universidad latinoamericana hacia el final del milenio, México: CRIM-UNAM.

14. Silva Herzog, J. (1999) Una historia de la universidad de México y sus problemas, México: Siglo XXI.

15. Tünnermann Bernheim, C. (1997) La Reforma Universitaria de Córdoba, en Temas de Hoy en la Educación Superior, no. 24,México: ANUIES.

16. Villaseñor, G. (coord.) (1997) La identidad en la educación superior en México, México: CESU/ UAM/ UNAM/ Universidad autónoma de Querétaro.

17. Yurén Camarena, M. T. (1997) "Razón de ser y sentido de la Universidad pública" en Villaseñor Guillermo (coord.) (1997) La identidad en la educación superior en México, México: CESU/ UAM/ UNAM/ Universidad autónoma de Querétaro. 